\title{
Unveiling healthy carriers and subclinical infections among household contacts of leprosy patients who play potential roles in the disease chain of transmission
}

\author{
Sérgio Araújo1, Janaína Lobato ${ }^{1,2}$, Érica de Melo Reis ${ }^{1,3}$, \\ Dulcinéa Oliveira Bernardes Souza', Maria Aparecida Gonçalves', \\ Adeilson Vieira Costa ${ }^{1}$, Luiz Ricardo Goulart ${ }^{1,3,4 /+}$, Isabela Maria Bernardes Goulart ${ }^{1}$
}

${ }^{1}$ Centro Nacional de Referência para Hanseníase e Dermatologia Sanitária, Hospital das Clínicas, Faculdade de Medicina
${ }^{2}$ Instituto de Ciências Biomédicas ${ }^{3}$ Instituto de Bioquímica e Genética, Universidade Federal de Uberlândia, Uberlândia, MG, Brasil
${ }^{4}$ Department of Medical Microbiology and Immunology, Genome and Biomedical Sciences Facility, University of California, Davis, CA, USA

Leprosy transmission still occurs despite the availability of highly effective treatment. The next step towards successfully eliminating leprosy is interrupting the chain of transmission of the aetiological agent, Mycobacterium leprae. In this investigation, we provide evidence that household contacts (HHCs) of leprosy patients might not only have subclinical infections, but may also be actively involved in bacilli transmission. We studied 444 patients and 1,352 contacts using anti-phenolic glycolipid-I (PGL-I) serology and quantitative polymerase chain reaction (qPCR) to test for M. leprae DNA in nasal swabs. We classified the patients according to the clinical form of their disease and the contacts according to the characteristics of their index case. Overall, $63.3 \%$ and $34.2 \%$ of patients tested positive by ELISA and PCR, respectively. For HHCs, $13.3 \%$ had a positive ELISA test result and $4.7 \%$ had a positive PCR test result. The presence of circulating anti-PGL-I among healthy contacts (with or without a positive PCR test result from nasal swabs) was considered to indicate a subclinical infection. DNA detected in nasal swabs also indicates the presence of bacilli at the site of transmission and bacterial entrance. We suggest that the concomitant use of both assays may allow us to detect subclinical infection in HHCs and to identify possible bacilli carriers who may transmit and disseminate disease in endemic regions. Chemoprophylaxis of these contacts is suggested.

Key words: leprosy - epidemiology - nasal swabs - anti-PGL-I serology - PCR - M. leprae

Leprosy is one of the oldest and most devastating diseases to affect humans. Its aetiological agent is $M y$ cobacterium leprae, an obligate intracellular pathogen with tropism for macrophages and Schwann cells.

M. leprae bacilloscopy is the gold standard laboratory assay for determining the presence of the disease and its operational classification (OC) for treatment purposes (WHO 1982). Ridley-Jopling's classification of clinical forms (CFs) (Ridley \& Jopling 1966) includes a broad spectrum of immune responses to $M$. leprae. These responses are further subdivided into two operational classifications: paucibacillary (PB) patients [tuberculoid (TT) and borderline tuberculoid (BT) leprosy forms], characterised by a strong delayed hypersensitivity to the Mitsuda test, and multibacillary (MB) patients [borderline-boderline (BB), borderline lepromatous (BL) and lepromatous (LL) leprosy $\mathrm{CFs}$ ], characterised by high antibody titres and decreasing degrees of cell-mediated immune responses that approach anergy (Hastings et al. 1988).

The current multidrug therapy treatment originated 30 years ago (WHO 1982). A decade later, the resolu-

Financial support: FAPEMIG, CNPq, CAPES, FUNASA/MS Brasil + Corresponding author: 1rgoulart@ucdavis.edu

Received 28 March 2012

Accepted 18 July 2012 tion of the World Health Organization (WHO) to eliminate leprosy as a public health problem (WHO 1991) has significantly increased worldwide access to treatment and has contributed to a remarkable reduction in the prevalence of the disease. However, the current global prevalence of leprosy is estimated at 192,246 cases, with 226,474 new cases detected in 2010 (WHO 2011). The data indicate that active transmission still occurs, despite the availability of highly effective treatment. Brazil, the second most affected country in the world, accounted for over $92 \%$ of the 37,740 new cases detected in the Americas in 2010 (WHO 2011).

The upper respiratory tract is considered the main portal for the entry and exit of M. leprae (Patrocínio et al. 2005, Martinez et al. 2011) and individuals with active disease (MB patients in particular) are the main sources of infection (Job et al. 2008). Because M. leprae is an obligate intracellular pathogen and there is little evidence of non-human reservoirs in endemic countries, it is most likely that leprosy spreads from person to person. Healthy carriers and people with subclinical infection have been recognised as links in the chain of M. leprae transmission (Hatta et al. 1995, Beyene et al. 2003).

We have demonstrated that molecular diagnostics and immunology are useful for understanding leprosy and for proposing actions that will successfully eliminate the disease, aiming for a detection rate of 10 new cases per 100,000 inhabitants (Brazilian Disease Notification System - dtr2004.saude.gov.br/sinanweb/tabnet/dh?sinannet/ 
hanseniase/bases/Hansbrnet.def). Based on the presence of M. leprae DNA in nasal swabs, we were able to identify possible transmitters and by detecting anti-phenolic glycolipid-I (PGL-I) in circulation, we identified subclinical infection among household contacts (HHCs). Both of these advances may significantly impact leprosy monitoring programs.

\section{SUBJECTS, MATERIALS AND METHODS}

Study population - HHCs were recruited at the National Reference Centre for Sanitary Dermatology and Leprosy (CREDESH), state of Minas Gerais, Brazil. The research was approved by the Federal University of Uberlândia Research Ethical Committee (025/2000) and all participants signed an informed consent form and authorised the collection of the samples. CREDESH is a public health care facility in an endemic region where routine prevention, including BCG vaccination, HHCs monitoring, early case detection and treatment are available and are under constant supervision.

Treatment-naïve leprosy patients (LPs) $(n=444)$ were diagnosed by a committee of experts based on clinical and laboratory tests and were classified according to $\mathrm{OC}$ (PB or MB) and to the CF of their disease (TT, BT, BB, $\mathrm{BL}$ or LL). A classification for indeterminate (I) forms of leprosy was also added.

HHCs $(n=1,352)$ who resided with LPs or had resided with LPs in the five years prior to diagnosis were examined by specialised physicians for signs or symptoms that were suggestive of leprosy. They were classified according to the $\mathrm{OC}$ and the $\mathrm{CF}$ of their index case (IC).

Bacillary index (BI) - Slit-skin smears from the patients' left and right ear lobes, elbows, knees and lesions were evaluated for the presence of acid-fast bacilli (AFB) with optical microscopy using Ziehl-Neelsen staining. The samples with observed AFB were considered positive.

Mitsuda test - An intradermal injection of $0.1 \mathrm{~mL}$ of Mitsuda, a heat-killed M. leprae suspension of $6 \times 10^{7}$ bacilli/mL produced by the Lauro de Souza Lima Institute, was given in the upper forearm. After 21 days, a positive late reaction (Mitsuda test) was characterised by a nodular epithelioid granuloma larger than $3 \mathrm{~mm}$ in diameter.

ELISA anti PGL-I serology - To test for IgM antibodies in sera, an indirect ELISA to detect antibodies against the $M$. leprae native PGL-I molecule (donated by Dr John Spencer, Colorado State University, USA) was performed using methodology that has been previously described (Lobato et al. 2011).

DNA extraction and polymerase chain reaction $(P C R)$ - Nasal swabs were collected by swabbing small flexible brushes into the nasal septa of both nasal fosses. The swab stems with samples were deposited in sterile tubes containing $500 \mu \mathrm{L}$ of lysis buffer $(\mathrm{NaCl} 400 \mathrm{mM}$,

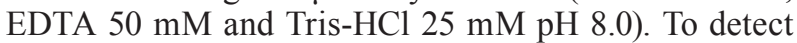
$M$. leprae DNA in nasal swab samples, a pair of primers targeting the RLEP3 region (X17153) of the M. leprae genome, were used to amplify a 130-bp fragment, using standardised conditions for PCR reactions, as described elsewhere (Goulart et al. 2007).
Statistical analysis - Descriptive analyses of the data were conducted. Pearson's correlation coefficient and linear and non-linear regressions were used to determine the association between the results of the assays across OCs and CFs. Fisher's exact test (2-tailed) was used to test the association between the ELISA and the PCR positivity results for contacts in a $2 \times 2$ contingency table. Statistical significance was set at $\mathrm{p}<0.05$.

\section{RESULTS}

$L P S$ - The percentages of positive results from the anti PGL-I ELISA and the M. leprae DNA detection using nasal swabs from the patients are listed in Table I and are classified according to the CF of patients' disease. Among LPs $(\mathrm{n}=444), 29.7 \%$ (132) were PB patients and 70.3\% (312) were MB patients. The percentages of patients with each CF were $1.1 \%$ (5) I, $10.1 \%$ (45) TT, 38.7\% (172) BT, $16.7 \%$ (74) BB, $14.2 \%$ (63) BL and 19.1\% (85) LL.

All PB patients were BI-negative, whereas $76 \%$ (237/312) of the MB patients were BI-positive. The distribution of BI-positive patients according to the CF of their disease was as follows: $0 \%$ for I and TT, 9.3\% (16/172) for BT, $98.6 \%$ (73/74) for BB and $100 \%$ for BL (63) and LL (85). A strong positive correlation was observed between the BI and CF [Pearson $\mathrm{r}=0.8826,95 \%$ confidence interval (CI) 0.0012-0.9922, $\left.\mathrm{p}=0.0474, \mathrm{R}^{2}=0.7790\right]$.

The Mitsuda test results for the 116 patients who were taking corticosteroids or receiving other interfering treatments were excluded from the analysis. Among the 328 patients with reliable results, $34.8 \%$ (115) were PB patients and, within this group, $9.6 \%$ (11) were negative and $90.4 \%$ (104) were positive. The prevalence of positive results among MB patients was $19.3 \%$ (41/213). The distribution of the patients who tested positive on the Mitsuda test, according to the CF of their disease, was as follows: $80 \%$ (4/5) for I, $100 \%$ (40/40) for TT, $71.9 \%(97 / 135)$ for BT, $7 \%$ (3/43) for BB, $2.6 \%(1 / 38)$ for BL and none for LL (67). A strong negative correlation between the Mitsuda test and the $\mathrm{CF}$ of the disease was observed (Pearson $\mathrm{r}=-0.9163$, $95 \%$ CI -0.9946 to $-0.1773, \mathrm{p}=0.0287, \mathrm{R}^{2}=0.8396$ ).

There was an inversely proportional relationship between the percentages of patients who tested positive on the BI and the Mitsuda test. The best-fitting curve for the percentages of patients with positive results on the BI was a positive logarithmic regression and for the Mitsuda test, it was a negative logarithmic regression, both towards the MB forms (BB, BL and LL) (Figure).

The prevalence of positive results among MB patients was $83 \%$ (256/312), but a similar percentage of PB patients, 83.3\% (110/132), tested negative. A strong positive correlation was observed between the anti PGL-I ELISA and the CF of the disease (Pearson $\mathrm{r}=0.9210$, 95\% CI 0.2063-0.9949, $\mathrm{p}=0.0263, \mathrm{R}^{2}=0.8482$ ).

The overall prevalence of positive test results using PCR amplification to detect $M$. leprae DNA in nasal swabs was $34.2 \%$ (152/444). The prevalence of positivity among PB patients was 5.3\% (7/132) and among the MB patients, it was $46.5 \%(145 / 312)$. A strong positive correlation between the PCR for M. leprae DNA detection and the $\mathrm{CF}$ of the disease was observed (Pearson $\mathrm{r}=0.9693$, $95 \%$ CI 0.6008-0.9981, $\mathrm{p}=0.0064, \mathrm{R}^{2}=0.9396$ ). 
TABLE I

Positivity of anti-phenolic glycolipid-I (PGL-I) ELISA serology and Mycobacterium leprae DNA in nasal swabs detected by polymerase chain reaction (PCR)

in patients and their household contacts according to their clinical forms and their index case (IC), respectively

\begin{tabular}{|c|c|c|c|c|c|}
\hline \multirow[b]{2}{*}{ Patient clinical classification } & \multicolumn{2}{|c|}{ Anti PGL-I ELISA } & \multicolumn{2}{|c|}{ Nasal swab PCR } & \multirow[b]{2}{*}{$\begin{array}{l}\text { Total } \\
\text { n (\%) }\end{array}$} \\
\hline & $\begin{array}{c}\text { Negative } \\
\mathrm{n}(\%)\end{array}$ & $\begin{array}{c}\text { Positive } \\
\text { n (\%) }\end{array}$ & $\begin{array}{c}\text { Negative } \\
\mathrm{n}(\%)\end{array}$ & $\begin{array}{c}\text { Positive } \\
\text { n (\%) }\end{array}$ & \\
\hline $\mathrm{I}$ & $5(100)$ & $0(0)$ & $4(80)$ & $1(20)$ & $5(1.1)$ \\
\hline $\mathrm{TT}$ & $40(88.9)$ & $5(11.1)$ & $43(95.6)$ & $2(4.4)$ & $45(10.1)$ \\
\hline BT & $106(61.6)$ & $66(38.4)$ & $162(94.2)$ & $10(5.8)$ & $172(38.7)$ \\
\hline BB & $7(9.5)$ & $67(90.5)$ & $49(66.2)$ & $25(33.8)$ & $74(16.7)$ \\
\hline $\mathrm{BL}$ & $3(4.8)$ & $60(95.2)$ & $27(42.9)$ & $36(57.1)$ & $63(14.2)$ \\
\hline LL & $2(2.4)$ & $83(97.6)$ & $7(8.2)$ & $78(91.8)$ & $85(19.2)$ \\
\hline Total & $163(36.7)$ & $281(63.3)$ & $292(65.8)$ & $152(34.2)$ & $444(100)$ \\
\hline \multicolumn{6}{|l|}{ Clinical classification of IC } \\
\hline I & $7(77.8)$ & $2(22.2)$ & $9(100)$ & $0(0)$ & $9(0.7)$ \\
\hline TT & $93(86.9)$ & $14(13.1)$ & $103(96.3)$ & $4(3.7)$ & 107 (7.9) \\
\hline BT & $365(88.6)$ & 47 (11.4) & $396(96.1)$ & $16(3.9)$ & $412(30.5)$ \\
\hline $\mathrm{BB}$ & $214(87.7)$ & $30(12.3)$ & $230(94.3)$ & $14(5.7)$ & $244(18)$ \\
\hline $\mathrm{BL}$ & $210(86.8)$ & $32(13.2)$ & $225(93)$ & $17(7)$ & $241(17.8)$ \\
\hline LL & $283(83.7)$ & $55(16.3)$ & $326(96.4)$ & $12(3.6)$ & $339(25.1)$ \\
\hline Total & $1,172(86.7)$ & $180(13.3)$ & $1,289(95.3)$ & $63(4.7)$ & $1,352(100)$ \\
\hline
\end{tabular}

BB: borderline-borderline; BL: borderline lepromatous; BT: borderline tuberculoid; I: indeterminate; LL: lepromatous leprosy; TT: tuberculoid.

Both assays, the anti PGL-I ELISA and the PCR to detect $M$. leprae DNA, yielded more positive results among the MB forms of the disease (BB, BL and LL). A geometrical regression provided the best fit for representing the percentages of positive results for anti PGL-I antibodies and a linear regression provided the best fit for the results of the M. leprae DNA detection (Figure).

HHCs - Regarding the OC of the IC, 22.4\% (303) of the HHCs had a PB IC and $77.6 \%(1,049)$ of the HHCs had an MB IC. The distribution of HHCs according to the CF of their IC is also shown in Table I.

The prevalence of positive results to the anti PGL-I ELISA by the OC of the IC were $11.5 \%(35 / 303)$ for PB contacts and $13.8 \%(145 / 1,049)$ for MB contacts. A strong positive correlation was observed between a positive response to the anti PGL-I ELISA and the CF of the IC (Pearson $\mathrm{r}=0.9163,95 \%$ CI $0.1775-0.9946, \mathrm{p}=$ $0.0287, \mathrm{R}^{2}=0.83970$ ).

The prevalence of positive results using PCR amplification to detect $M$. leprae DNA by the OC of the IC were $3.3 \%(10 / 303)$ for PB contacts and 5.1\% $(53 / 1,049)$ for $\mathrm{MB}$ contacts. There was no statistically significant relationship between the detection of M. leprae DNA and the CF of the IC ( $p>0.05)$.

A $2 \times 2$ contingency table is presented in Table II to compare the performance of the anti-PGL-I ELISA and the detection of M. leprae DNA in nasal swabs from contacts of LPs. A two-tailed Fisher's exact test indicated a significant association between the results from the PCR using nasal swabs and the results from the anti-PGL-I serology $(\mathrm{p}<0.0034)$. The anti-PGL-I and the PCR results alone yielded positive results for $13.3 \%$ and $4.7 \%$ subjects, respectively.

\section{DISCUSSION}

We have demonstrated that using anti-PGL-I ELISA serology and PCR detection of M. leprae DNA in nasal swabs to identify high-risk HHCs is a useful monitoring strategy. HHCs who are carriers or who have subclinical infection may be at risk of disseminating bacilli and maintaining the chain of leprosy transmission. The result of each test alone has special clinical significance. The anti-PGL-I serology was strongly correlated with the MB $\mathrm{OC}$ and associated CFs of leprosy, indicating a possible active infection, as shown elsewhere (Cho et al. 2001, Martinez et al. 2011). However, the presence of M. leprae DNA in nasal swabs has been used as an epidemiological tool to understand the role of both patients and contacts in the complex transmission chain (Hatta et al. 1995, Beyene et al. 2003, Patrocínio et al. 2005, Job et al. 2008).

Among HHCs of LPs, $4.7 \%$ tested positive for $M$. leprae DNA in nasal swabs, similar to the percentages of 


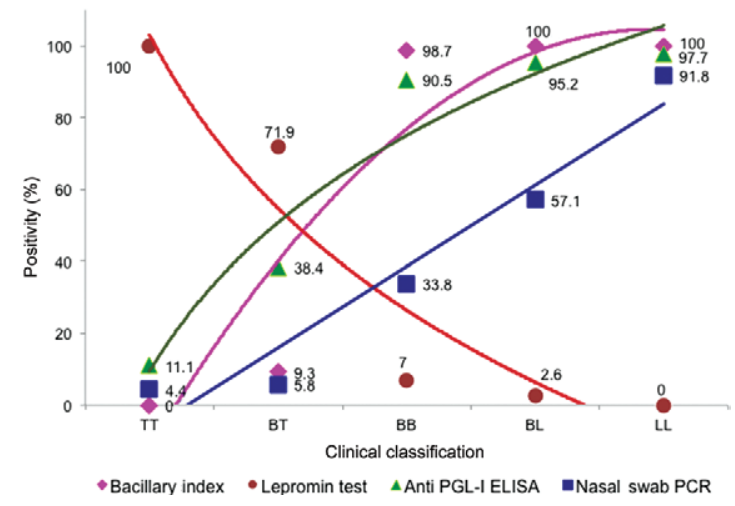

Tendency lines for the positivity (\%) of bacilloscopic indexes, Mitsuda test, anti-phenolic glycolipid-I (PGL-I) ELISA serology and $M y$ cobacterium leprae DNA in nasal swab detected by polymerase chain reaction (PCR) in leprosy patients according to their clinical forms. BB: borderline-borderline; BL: borderline lepromatous; BT: borderline tuberculoid; LL: lepromatous leprosy; TT: tuberculoid.

positive results observed among TT or BT patients, but only half of the percentage observed in nasal biopsies of PB patients described elsewhere (Patrocínio et al. 2005). This previous finding led us to the following hypothesis regarding the infection route: the bacillus invades the mucosa, passing through the nasal inferior turbinate and through the peripheral blood (Patrocínio et al. 2005), eventually reaching Schwann cells and the mononuclear phagocytic system (Harboe 1985). Therefore, these findings suggest that contacts may be exposed to a source of infection who harbours bacilli in the nose even for a short period of time because nasal biopsies detect $14 \%$ more positive test results than do nasal swabs (Patrocínio et al. 2005). Due to the complex relationships among genetic, immunological and environmental factors, most of the contacts will not become infected or develop leprosy (Goulart \& Goulart 2009). However, they may spread $M$. leprae to susceptible individuals and this may be a plausible mode of transmission. Our results also corroborate previous studies that have detected $M$. leprae DNA in the nasal mucosa of endemic populations (Hatta et al. 1995, Beyene et al. 2003, Martinez et al. 2011), regardless of the OC and CFs of their ICs.

Our results revealed a linear correlation between bacillary DNA levels and the CFs of the patients' diseases. This response was positively correlated with the anti-PGL-I profile and was negatively correlated with the Mistuda test across CFs. These relationships further support and reinforce our hypothesis that the nasal mucosa acts as both the site of entry and of transmission. This assumption is further supported by the HHCs data, in which $13 \%$ tested positive for IgM anti-PGL-I, a higher percentage than that observed in TT patients, with an increasing prevalence of positive results for MB forms of leprosy. These results further corroborate other studies that demonstrated anti-PGL-I seropositivity is a high risk factor for the development of disease in HHCs, mainly contacts of MB ICs (Douglas et al. 2004, Goulart et al. 2008).
TABLE II

Contingency table for the anti-phenolic glycolipid-I (PGL-I)

ELISA serology and Mycobacterium leprae DNA in nasal swabs detected by polymerase chain reaction (PCR) from contacts of leprosy patients

\begin{tabular}{lccc}
\hline & \multicolumn{2}{c}{ Anti PGL-I ELISA } & \\
\cline { 2 - 3 } & $\begin{array}{c}\text { Negative } \\
\text { Nasal swab PCR }\end{array}$ & $\begin{array}{c}\text { Positive } \\
\mathrm{n}(\%)\end{array}$ & $\begin{array}{c}\text { Total } \\
\mathrm{n}(\%)\end{array}$ \\
\hline Negative & $1,126(83.3)$ & $163(12)$ & $1,289(95.3)$ \\
Positive & $46(3.4)$ & $17(1.3)$ & $63(4.7)$ \\
\hline Total & $1,172(86.7)$ & $180(13.3)$ & $1,352(100)$ \\
\hline
\end{tabular}

The presence of M. leprae DNA in the nasal mucosa of HHCs of LPs reflects the presence of bacilli in the nose, which may become a source of infection or transmission. However, the presence of M. leprae DNA in nasal secretions does not determine infection; it only indicates nasal carriage, which may imply an active leprosy transmission. Further investigation of anti-PGL-I seropositivity indicates that bacilli have successfully entered the circulatory system and may indicate subclinical infection. Moreover, the number of seropositive patients does not indicate the true prevalence of infected contacts because PB patients rarely produce specific antibodies, suggesting that the number of infected contacts might be underestimated.

HHCs comprise a recognisable group of individuals with a high risk of disease, as they live in close proximity to a source of infection (Sales et al. 2011). Their participation in the dissemination and spread of M. leprae to susceptible persons in endemic communities cannot be neglected. The observed distribution of positive test results among HHCs of ICs representing both OCs and every $\mathrm{CF}$ of the disease indicates that they all experience bacillary pressure, that they are susceptible to infection due to continuous interaction with a source of infection and that $M$. leprae is widely present among them.

Finally, a successful leprosy control program requires prevention, early diagnosis, treatment and interruption of the chain of transmission. Therefore, we reinforce our previous suggestion (Goulart et al. 2008), which proposes chemoprophylaxis of HHCs with subclinical infection as a prevention strategy. Additionally, we propose the treatment of healthy and contact carriers to achieve bacilli clearance and to interrupt leprosy transmission.

\section{ACKNOWLEDGEMENTS}

To all the staff of the National Reference Centre in Leprosy of the Federal University of Uberlândia, for fundamental support, and to Josiela Alves Gonçalves and Juliano Araújo, for the software's data management.

\section{REFERENCES}

Beyene D, Aseffa A, Harboe M, Kidane D, Macdonald M, Klatser PR, Bjune GA, Smith WC 2003. Nasal carriage of Mycobacterium leprae DNA in healthy individuals in Lega Robi village, Ethiopia. Epidemiol Infec 131: 841-848. 
Cho SN, Cellona RV, Villahermosa LG, Fajardo Jr TT, Balagon MV, Abalos RM, Tan EV, Walsh GP, Kim JD, Brennan PJ 2001. Detection of phenolic glycolipid 1 of Mycobacterium leprae in sera from leprosy patients before and after start of multidrug therapy. Clin Diagn Lab Immunol 8: 138-142.

Douglas JT, Cellona RV, Fajardo Jr TT, Abalos RM, Balagon MV, Klatser PR 2004. Prospective study of serological conversion as a risk factor for development of leprosy among household contacts. Clin Diagn Lab Immunol 11: 897-900.

Goulart IM, Bernardes Souza DO, Marques CR, Pimenta VL, Gonçalves MA, Goulart LR 2008. Risk and protective factors for leprosy development determined by epidemiological surveillance of household contacts. Clin Vaccine Immunol 15: 101-105.

Goulart IM, Cardoso AM, Santos MS, Gonçalves MA, Pereira JE, Goulart LR 2007. Detection of Mycobacterium leprae DNA in skin lesions of leprosy patients by PCR may be affected by amplicon size. Arch Dermatol Res 299: 267-271.

Goulart IMB, Goulart LR 2009. Leprosy pathogenetic background: a review and lessons from other mycobacterial diseases. Arch Dermatol Res 301: 123-137.

Harboe M 1985. The immunology of leprosy. In RC Hastings, Leprosy, Churchill Livingstone, Edinburgh, p. 53-87.

Hastings RC, Gillis TP, Krahenbuhl JL, Franzblau SG 1988. Leprosy. Clin Microbiol Rev 1: 330-348.

Hatta M, van Beers SM, Madjid B, Djumadi A, de Wit MY, Klatser PR 1995. Distribution and persistence of Mycobacterium leprae nasal carriage among a population in which leprosy is endemic in Indonesia. Trans R Soc Trop Med Hyg 89: 381-385.
Job CK, Jayakumar J, Kearney M, Gillis TP 2008. Transmission of leprosy: a study of skin and nasal secretions of household contacts of leprosy patients using PCR. Am J Trop Med Hyg 78: 518-521.

Lobato J, Costa MP, Reis EM, Gonçalves MA, Spencer JS, Brennan PJ, Goulart LR, Goulart IMB 2011. Comparison of three immunological tests for leprosy diagnosis and detection of subclinical infection. Lepr Rev 82: 1-14.

Martinez TS, Figueira MM, Costa AV, Gonçalves MA, Goulart LR, Goulart IM 2011. Oral mucosa as a source of Mycobacterium leprae infection and transmission and implications of bacterial DNA detection and the immunological status. Clin Microbiol Infec 17: 1653-1658.

Patrocínio LG, Goulart IM, Goulart LR, Patrocínio JA, Ferreira FR, Fleury RN 2005. Detection of Mycobacterium leprae in nasal mucosa biopsies by the polymerase chain reaction. FEMS Immunol Med Microbiol 44: 311-316.

Ridley DS, Jopling WH 1966. Classification of leprosy according to immunity: a five group system. Int J Lepr Other Mycobact Dis 34: $255-273$.

Sales AM, Leon AP, Dupree NC, Hacker MA, Nery JAC, Sarno EN, Penna MLF 2011. Leprosy among patient contacts: a multilevel study of risk factors. PLoS Negl Trop Dis 5: e1013.

WHO - World Health Organization 1982. Chemotherapy of leprosy for control programmes. WHO Tech Rep Ser 675: 1-33.

WHO - World Health Organization 1991. Leprosy Resolution World Health Assembly 44.9. Forty-fourth World Health Assembly, WHO, Available from: paho.org/english/ad/dpc/cd/lep-wha44.9.htm.

WHO - World Health Organization 2011. Leprosy update. Wkly Epidemiol Rec 36: 389-400. 